\title{
Associação do polimorfismo IL-6 -174G > C (rs1800795) com escoliose idiopática da adolescência: Evidências de um estudo de caso-controle e metanálise
}

\section{Association of the IL-6 -174G > C (rs1800795) Polymorphism with Adolescent Idiopathic Scoliosis: Evidence from a Case-Control Study and Meta-Analysis}

\author{
Mohammad Reza Sobhan ${ }^{1} \quad$ Masoud Mahdinezhad-Yazdi ${ }^{1}$ \\ Kazem Aghili $^{3}$ Hossein Neamatzadeh ${ }^{4,5}$ \\ ${ }^{1}$ Department of Orthopedics, Shahid Sadoughi University of Medical \\ Sciences, Yazd, Iran \\ 2 Department of Medical Genetics, School of Medicine, Shiraz \\ University of Medical Sciences, Shiraz, Iran \\ ${ }^{3}$ Department of Radiology, Shahid Sadoughi University of Medical \\ Sciences, Yazd, Iran \\ ${ }^{4}$ Department of Medical Genetics, Shahid Sadoughi University of \\ Medical Sciences, Yazd, Iran \\ ${ }^{5}$ Mother and Newborn Health Research Center, Shahid Sadoughi \\ University of Medical Sciences, Yazd, Iran \\ Rev Bras Ortop 2020;55(1):17-26.
}

Seyed Alireza Dastgheib ${ }^{2}$ Hossein Ahrar $^{3}$

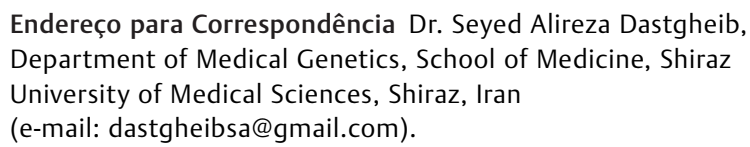
(e-mail: dastgheibsa@gmail.com).

\section{Resumo}

Palavras-chave

- escoliose idiopática

- interleucina-6

- polimorfismo

- associação

- metanálise
Estudos epidemiológicos recentes identificaram que o polimorfismo -174G $>C$ (rs1800795) na região promotora do gene interleucina-6 (IL-6) está associado ao risco de desenvolver escoliose idiopática da adolescência (EIA), mas apresentaram resultados inconsistentes e controversos. Assim, realizamos um estudo de caso-controle e metanálise para obter uma estimativa mais precisa da relação entre o polimorfismo IL-6 $-174 \mathrm{G}>\mathrm{C}$ e o risco de desenvolver EIA. Um total de 80 pacientes com EIA e 80 controles saudáveis pareados foram genotipados usando o ensaio de reação em cadeia de polimerase de polimorfismos de comprimento de fragmentos de restrição (RCP-PCFR). Além disso, todos os estudos elegíveis publicados até junho de 2018 foram identificados por meio de uma pesquisa nas bases de dados PubMed, EMBASE, Google Scholar e China National Knowledge Infrastructure (CNKI). Calculamos as razões de probabilidades (RPs) e os intervalos de confiança de 95\% (ICs95\%) para avaliar a associação. Um total de 10 estudos elegíveis compreendendo 1.695 casos de EIA e 2.097 controles saudáveis foram incluídos na metanálise. Os dados agrupados sugeriram uma associação significativa entre o polimorfismo IL-6 -174G >C e a suscetibilidade a desenvolver EIA que foi demonstrada em quatro modelos genéticos, ou seja, alélico ( $C$ versus $\mathrm{G} ; \mathrm{RP}=0,671$; IC95\%: 0,457-0,985; $p=0,042$ ), heterozigótico (GC versus GG; RP $=0,734$; IC95\%: 0,554-0,973; $p=0,032)$, dominante $(C C+G C$ versus $G G ; R P=0,660$; IC95\%: $0,440-0,990 ; p=0,044$ ) e recessivo (CC versus $C G+G G$; $R P=0,506$; IC95\%: recebido

04 de Setembro de 2018 aceito

27 de Novembro de 2018
DOI https://doi.org/ 10.1055/s-0039-1700813. ISSN 0102-3616.
Copyright $\odot 2020$ by Sociedade Brasileira License terms de Ortopedia e Traumatologia. Published by Thieme Revinter Publicações Ltda, Rio de Janeiro, Brazil 


\begin{abstract}

\section{Keywords}

- idiopathic scoliosis

- interleukin-6

- polymorphism

- association

- meta-analysis

Recent epidemiological studies have identified that the $-174 \mathrm{G}>\mathrm{C}$ (rs1800795) polymorphism in the promoter region of the interleukin-6 (IL-6) gene is associated with the risk of developing adolescent idiopathic scoliosis (AIS), but they presented inconsistent and controversial results. Thus, we performed a case-control study and meta-analysis to derive a more precise estimation of the relationship between the IL- $6-174 \mathrm{G}>$ C polymorphism and the risk of developing AIS. A total of 80 patients with AIS and 80 matched healthy control subjects were genotyped using the polymerase chain reaction-restriction fragment length polymorphism (PCR-RFLP) assay. In addition, all eligible studies published up to June 2018 were identified through a search in the PubMed, EMBASE, Google Scholar, and China National Knowledge Infrastructure (CNKI) databases. We calculated the odds ratios (ORs) and $95 \%$ confidence intervals $(95 \% \mathrm{Cls})$ to assess the association. A total of 10 eligible studies comprising 1,695 AIS cases and 2,097 healthy controls were included in the meta-analysis. The pooled data suggested a significant association between the IL-6 -174G $>C$ polymorphism and the susceptibility to develop AIS, which was demonstrated under 4 genetic models, that is, the allelic ( $C$ versus $\mathrm{G}$; $\mathrm{OR}=0.671 ; 95 \% \mathrm{Cl}: 0.457-0.985 ; p=0.042$ ), heterozygous (CG versus GG; $\mathrm{OR}=0.734$; $95 \% \mathrm{Cl}$ : $0.554-0.973 ; p=0.032$ ), dominant $(C C+C G$ versus GG; OR $=0.660 ; 95 \% \mathrm{Cl}: 0.440-0.990 ; p=0.044)$ and recessive models (CC versus CG + GG; OR $=0.506 ; 95 \% \mathrm{Cl}: 0.264-0.970 ; p=0.040$ ). The stratification analysis by ethnicity revealed an increased risk of developing AIS in Caucasians, but not in Asians. The present meta-analysis, which is inconsistent with the previous meta-analysis, suggests that the IL-6 -174G > C polymorphism may increase the individual susceptibility to develop AIS, especially in Caucasians, and it could serve as a biomarker to predict the population at high risk of developing AIS.
\end{abstract}

0,264-0,970; $p=0,040)$. A análise de estratificação por etnia revelou um aumento do risco de desenvolver EIA em caucasianos, mas não em asiáticos. Esta metanálise, que é inconsistente com relação à metanálise anterior, sugere que o polimorfismo IL-6 $-174 \mathrm{G}>\mathrm{C}$ pode aumentar a suscetibilidade individual para desenvolver EIA, especialmente em caucasianos, e pode servir como um biomarcador para prever a população com alto risco de desenvolver EIA.

\section{Introdução}

A escoliose idiopática (EI) é o tipo mais comum de deformidade musculoesquelética, e afeta $\sim 3 \%$ das crianças e adolescentes. ${ }^{1,2}$ Escoliose refere-se a desvio da coluna vertebral superior a 10 graus no plano coronal, que é mais frequentemente descoberto por meio de programas de triagem escolar ou pelos pais. ${ }^{1,3} \mathrm{~A}$ progressão da El ocorre em três dimensões, com a coluna curvando-se simultaneamente em direção aos braços, e uma progressão de $\sim 10 \%$ para uma curva moderada ou grave. ${ }^{4,5}$ Existe uma alta variabilidade na manifestação clínica e no fenótipo de EI: ela pode surgir nas formas infantil (0-3 anos) e juvenil (4-9 anos), embora a maioria dos casos ocorra na população adolescente ( $\geq 10$ anos) de crianças saudáveis no geral, com início entre (ou próximo) a puberdade e a maturidade esquelética. ${ }^{6-8}$

A escoliose idiopática da adolescência (EIA) é prevalente em $1 \%$ a $3 \%$ dos adolescentes de 10 a 16 anos. ${ }^{6} O$ padrão de herança da EIA não é claro, porque muitos fatores, incluindo fatores genéticos, ambientais, o expossoma e suas interações combinadas, estão envolvidos. ${ }^{2,8}$ Estudos de associação genética de
EIA que foram realizados nos últimos anos, particularmente estudos de ligação, estudos de associação genômica ampla (EAGA) e estudos epigenéticos, identificaram vários loci gênicos, como LBX1, GPR126 SR1, ESR2, MATN1, POC5, IGF1 e VDR, associados à suscetibilidade a desenvolver EIA.,9-11 Eles demonstraram que alguns desses loci provavelmente contribuem para a suscetibilidade a desenvolver EIA, enquanto outros podem desempenhar um papel crítico na determinação da gravidade da curvatura da coluna vertebral e/ou para determinar se a curva é estável ou progressiva. ${ }^{9}$

A interleucina-6 (IL-6) é uma citocina pró-inflamatória e uma miocina anti-inflamatória que é rápida e transitoriamente produzida em reações a respostas imunes, reações inflamatórias e hematopoiese. ${ }^{12} \mathrm{O}$ gene humano IL-6 está localizado no cromossomo $7 \mathrm{p} 21-24$ com um promotor a montante contendo $303 \mathrm{bp}$, consistindo em 5 éxons e com um comprimento de $5 \mathrm{~kb} .{ }^{13-15}$ Recentemente, o polimorfismo do $-174 G>C$ (rs1800795) na região promotora do IL-6 tem sido investigado na patogênese da EIA, mas os resultados são controversos, o que pode ser devido ao pequeno tamanho das amostras e diferenças étnicas. Portanto, realizamos um estudo 
de caso-controle em pacientes lianianos com EIA, e uma metaanálise para estimar melhor o risco geral de desenvolver EIA causado pelo polimorfismo do IL-6 -174G $>$ C.

\section{Materiais e Métodos}

\section{Estudo de Caso-Controle}

\section{População do Estudo}

Todos os sujeitos forneceram consentimento livre e esclarecido antes do início do estudo, que foi aprovado pelo Comitê de Ética em Pesquisa Clínica do nosso instituto. No total, 80 pacientes consecutivos com EIA, que visitaram 7 clínicas ortopédicas em 6 cidades entre abril de 2014 e setembro de 2017, foram recrutados retrospectivamente para o presente estudo. Todos os pacientes foram submetidos a exames radiológicos usando radiografias posteroanteriores (PAs) em pé. Um total de 80 indivíduos saudáveis, pareados como grupo de controle, foram selecionados aleatoriamente a partir da população geral depois de confirmada a ausência de qualquer evidência de escoliose, curvatura da coluna vertebral ,ou outras condições ortopédicas de acordo com critérios radiográficos.

\section{Genotipagem}

Para a análise de genótipo, amostras de sangue periférico de todos os pacientes e controles foram coletadas, e o DNA genômico de cada amostra foi obtido usando um kit comercial (Cinnagen, Tehran, Iran). O polimorfismo do IL-6 -174G >C (rs1800795) foi genotipado usando a técnica de reação em cadeia de polimerase de polimorfismos de comprimento de fragmentos de restrição (RCP-PCFR). Para investigar o polimorfismo do IL-6 -174G > C (rs1800795), usamos os primers 5'TGACTTCAGCTTTACTCTTTGT-3' e reverso 5'-CTGATTGGAAACCTTATTAAG-3'. Os produtos da RCP foram digeridos com enzima de restrição Streptococcus faecalis ND547 (SfaNI) a $37^{\circ} \mathrm{C}$ até o dia seguinte, e depois, analisados por eletroforese em gel de agarose tingido a 2,0\% com brometo de etídio. O produto digerido da RCP gerou os seguintes fragmentos: homozigoto tipo selvagem (GG), três fitas constituídas por 140 bp e 58 bp; genótipo heterozigoto (GC), 198 bp, 140 bp e 58 bp; e genótipo mutante homozigoto (CC), uma fita de $198 \mathrm{bp}$.

\section{Análise Estatística}

As diferenças na distribuição de genótipos e alelos para pacientes com EIA e indivíduos controle foram avaliadas com o teste do Qui-quadrado $\left(\mathrm{X}^{2}\right)$. O equilíbrio de Hardy-Weinberg (EHW) foi computado com o teste qualidade de ajuste $X^{2}$ em nosso grupo de controle. Todas as análises estatísticas foram feitas usando o programa International Business Machines Statistical Package for the Social Sciences (IBM SPSS, IBM Corp., Armonk, NY, EUA), versão 20.0. Valores de $p<0,05$ foram bicaudais, e foram considerados sugestivos de associação.

\section{Metanálise}

\section{Estratégia de Pesquisa}

Para identificar todas as publicações que avaliaram a associação do polimorfismo IL-6 -174G > C (rs1800795) com a
EIA, realizamos uma pesquisa eletrônica abrangente nos bancos de dados PubMed, EMBASE, China National Knowledge Infrastructure (CNKI) e outras bases de dados chinesas de biomedicina até 15 de junho de 2018. Foi usada uma combinação dos seguintes termos do sistema de metadados Medical Subject Headings (MeSH) e palavras-chave: (adolescent idiopathic scoliosis OU scoliosis' OU AIS) E (interleukin-6 OU IL-6 OU -174G >C OU rs1800795) E (SNPs OU polymorphism OU genotype OU allele OU variation). A pesquisa foi limitada aos estudos em humanos publicados. Além disso, a lista de referências de estudos de caso-controle elegíveis e as revisões relacionadas foram pesquisadas manualmente para encontrar mais fontes em potencial.

\section{Critérios de Inclusão}

Os seguintes critérios de inclusão foram utilizados para selecionar a literatura para a metanálise: 1) estudos de caso-controle ou coorte; 2 ) estudos que avaliavam a associação do polimorfismo do IL-6 -174 G > C com a EIA; 3) estudos com dados suficientes para examinar uma razão de probabilidades (RP) com um intervalo de confiança de 95\% (IC95\%). Além disso, também foram utilizados os seguintes critérios de exclusão: 1) estudos que não eram de caso-controle ou de coorte; 2) estudos apenas com casos, sem controles; 3 ) estudos sem dados suficientes relatados; 4) resumos, comentários, relatos de casos, cartas, revisões, metanálises; e 5) duplicatas de publicações anteriores.

\section{Extração de Dados}

Dois autores extraíram, cuidadosa e independentemente, os dados de todos os estudos elegíveis de acordo com os critérios de inclusão listados anteriormente. As discordâncias foram resolvidas por meio de discussão entre os dois investigadores, ou um terceiro investigador foi consultado para resolver a disputa, e uma decisão final foi tomada por maioria dos votos. Para cada estudo incluído, foram coletadas as seguintes informações: primeiro autor, ano de publicação, país, etnia, número de casos e controles, métodos de genotipagem e evidência de EHW.

\section{Análise Estatística}

A força da associação do polimorfismo do IL-6 -174 G >C com a EIA foi avaliada usando a RP com o correspondente IC95\%. A significância das RPs agrupadas foi testada pelo teste $Z$, no qual $p<0,05$ foi considerado significativo. Os riscos (RPs) de desenvolver EIA associados ao polimorfismo do IL-6 -174 G > C foram estimados para cada estudo por cinco modelos genéticos: o modelo alélico ( $C$ versus $G$ ), o modelo homozigótico (CC versus GG), o modelo heterozigótico (CC versus GC), o modelo dominante (CC + GC versus GG), e o modelo recessivo (CC versus $G G+G C$ ). As estatísticas $Q$ e $\mathrm{I}^{2}$ foram utilizadas para avaliar a heterogeneidade do estudo na metanálise. Além disso, usamos a estatística $I^{2}$ para quantificar a heterogeneidade entre os estudos, que varia de $0 \%$ a $100 \%$, e representa a proporção da variabilidade entre os estudos atribuível à 
heterogeneidade, em vez de ao acaso. Um valor de $p$ menor do que 0,05 para a estatística $Q$ indicou presença de heterogeneidade entre os estudos, de forma que a estimativa da RP agrupada de cada estudo foi calculada pelo modelo de efeitos aleatórios (o método de DerSimonian e Laird). Caso contrário, o modelo de efeitos fixos (o método de Mantel-Haenszel) foi usado. Para cada estudo, examinamos se a distribuição de genótipos nos grupos controle estava de acordo com o EHW usando o teste $\mathrm{X}^{2}$. A análise de sensibilidade unidirecional, pela qual um único estudo da metanálise foi omitido por vez para refletir a influência do conjunto de dados individuais para a RP agrupada, foi realizada para avaliar a estabilidade dos resultados. Para detectar a presença de possível viés de publicação, foram usados a inspeção visual de Begg da simetria do gráfico de funil e o teste de regressão linear de Egger. Todos os testes estatísticos foram realizados utilizando o programa Comprehensive Meta-Analysis (CMA, Biostat, Englewood, NJ, EUA), versão 2.0. Todos os valores de $p$ na metanálise foram bilaterais, e valores menores do que 0,05 foram considerados significantivos.

\section{Resultados}

\section{Estudo de Caso-Controle}

A - Tabela 1 apresenta a distribuição de frequências de alelos e genótipos do polimorfismo do IL-6 -174G >C em casos de EIA e em indivíduos do grupo de controle. As distribuições do genótipo do polimorfismo do IL-6 -174G $>\mathrm{C}$ no grupo de controle estavam dentro do EHW $(p=0,818)$. As frequências do genótipo do polimorfismo (GG, GC e CC) do IL-6 -174G > C foram 92,50\%, 6,25\% e 1,25\% para pacientes com EIA, e de $95,0 \%, 5,0 \%$ e $0,0 \%$ para os indivíduos do grupo de controle, respectivamente. No presente estudo, não conseguimos encontrar associação estatisticamente significantiva entre o polimorfismo do IL-6 $-174 G>C$ e o risco de desenvolver EIA.

\section{Metanálises}

\section{Estudos Elegíveis}

Um fluxograma descrevendo o processo de seleção do estudo é apresentado na - Fig. 1. Após a exclusão de artigos duplicados e irrelevantes, um total de 10 estudos de casocontrole, ${ }^{14,16-23}$ incluindo 1.695 casos de EIA e 2.097 controles, foram selecionados para a metanálise. As características dos estudos elegíveis são apresentadas na - Tabela 2. Dos 10 estudos, $6^{16,17,21-23}$ foram realizados em populações asiáticas (1.331 casos de EIA e 1.324 controles), e $4^{14,18-20}$ foram realizados em populações caucasianas ( 364 casos de EIA e 773 controles). Três técnicas de genotipagem foram aplicadas nos estudos de caso-controle incluídos: RCP-PCFR, TaqMan e sequenciamento direto (SD). Todos os estudos indicaram que a distribuição de genótipos entre os controles era consistente com o EHW, a não ser por dois estudos (-Tabela 2 ).

\section{Síntese Quantitativa de Dados}

A - Tabela 3 resume os principais resultados da metanálise do polimorfismo IL-6 -174G > C e do teste de heterogeneidade. Os dados agrupados sugeriram uma associação significativa entre o polimorfismo do IL-6 -174G $>$ C e a suscetibilidade a desenvolver EIA por 4 modelos genéticos: alélico (C versus G: $R P=0,671 ; \quad I C 95 \%$ : 0,457-0,985; $p=0,042$; - Fig. 2A), heterozigótico (CG versus GG: RP $=0,734$; IC95\%: 0,554-0,973; $p=0,032$ ), dominante (CC+ CG versus GG: $\mathrm{RP}=0,660$; $\mathrm{IC} 95 \%$ : 0,440-0,990; $p=0,044$; -Fig. 2B) e recessivo (CC versus CG $+\mathrm{GG}$ : RP $=0,506$; IC95\%: 0,264-0,970; $p=0,040$ ).

A - Tabela 3 também lista os resultados das análises estratificadas por etnia, nas quais uma associação significativa entre o polimorfismo do IL-6 -174G > C e o risco de desenvolver EIA foi encontrada entre caucasianos em 3 modelos genéticos: alélico (C versus G: RP = 0,552; IC95\%: 0,318-0,959; $p=0,035$ ), homozigótico (CC versus GG: $\mathrm{RP}=0,329$; IC95\%:

Tabela 1 Distribuições de frequências de genótipos e alelos em casos de EIA e controles

\begin{tabular}{|l|l|l|l|l|}
\hline & Casos $(\boldsymbol{n}=\mathbf{8 0})$ & Controles $(\boldsymbol{n}=\mathbf{8 0})$ & RP (IC95\%) & Valor de $\boldsymbol{p}$ \\
\hline IL-6 -174 G $>$ C & & & & \\
\hline Genótipos & & & & \\
\hline GG & $74(92,50)$ & $76(95,0)$ & Referência & \\
\hline CG & $5(6,25)$ & $4(5,0)$ & $1,267(0,327-4,900)$ & 0,732 \\
\hline CC & $1(1,25)$ & $0(0,00)$ & $3,038(0,122-75,693)$ & 0,498 \\
\hline Alelo & & & & \\
\hline G & $153(95,62)$ & $156(97,50)$ & Referência & \\
\hline C & $7(4,38)$ & $4(2,50)$ & $1,784(0,512-6,219)$ & 0,363 \\
\hline Modelos genéticos & & & & 0,516 \\
\hline $\begin{array}{l}\text { Dominante } \\
\text { (CC+ CG versus GG) }\end{array}$ & & & $1,541(0,418-5,681)$ & \\
\hline $\begin{array}{l}\text { Recessivo } \\
\text { (CC versus CG + GG) }\end{array}$ & & & $3,038(0,122-75,693)$ & 0,498 \\
\hline
\end{tabular}

Abreviaturas: IC95\%, intervalo de confiança de 95\%; EIA, escoliose idiopática da adolescência; RP, razão de probabilidades. 


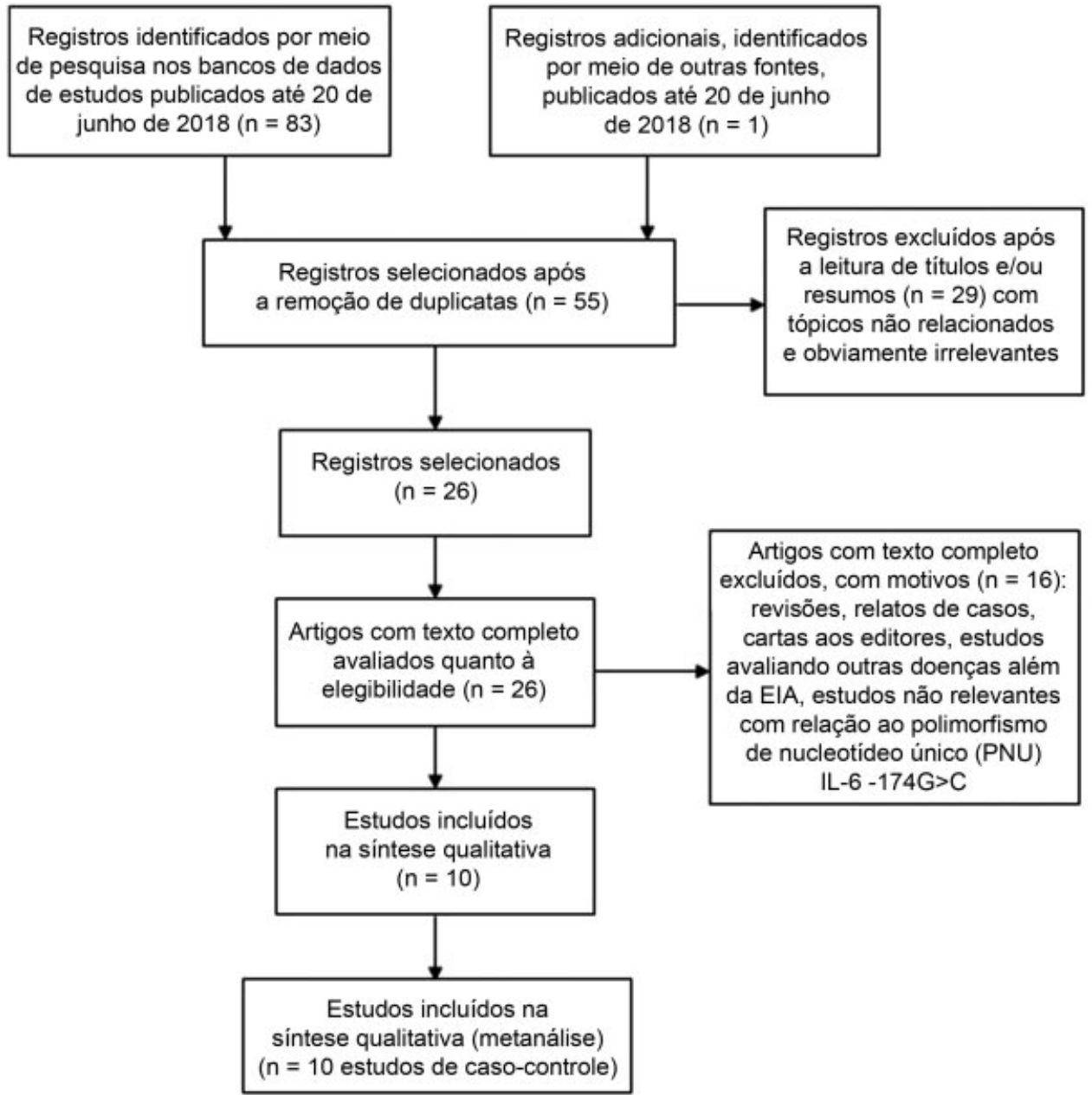

Fig. 1 Fluxograma da seleção de estudos individuais para a metanálise.

0,114-0,951; $p=0,040$ ), e recessivo (CC versus CG $+\mathrm{GG}$ : RP $=0,408$; IC95\%: 0,180-0,925; $p=0,032$ ), mas não em populações asiáticas. Além disso, realizamos análises agrupadas entre a população chinesa. Entretanto, os resultados mostraram que não houve associação estatisticamente significativa entre 0 polimorfismo do IL-6 -174G > C e o risco de desenvolver EIA nessa população chinesa ( - Tabela 2 ).

\section{Teste de Heterogeneidade}

A - Tabela 3 resume o resultado principal de heterogeneidade $(\mathrm{H})$ entre os estudos. Houve uma heterogeneidade significativa entre os estudos detectada em quatro modelos genéticos: alélico ( $C$ versus $G$ : $\mathrm{I}^{2}=73,65 ; p_{\mathrm{H}} \leq 0,001$ ), heterozigótico (CG versus GG: $\mathrm{I}^{2}=77,57 ; p_{\mathrm{H}} \leq 0,001$ ), dominante (CC + CG versus GG: $\left.\mathrm{I}^{2}=61,46 ; p_{\mathrm{H}}=0,008\right)$ e recessivo (CC versus $C G+G G: I^{2}=67,97 ; p_{H}=0,008$ ). Por isso, exploramos as possíveis fontes de heterogeneidade por análise estratificada por etnia. Os resultados mostraram que estudos em populações asiáticas foram fonte substancial de heterogeneidade. Além disso, a remoção dos estudos que desviavam do EHW não alterou significativamente a heterogeneidade significativa entre os estudos (dados não mostrados), indicando que os modelos eram robustos.

\section{Análise de Sensibilidade}

A análise de sensibilidade foi realizada para explorar o impacto de um estudo individual sobre as RPs agrupadas. Os resultados revelaram que nenhum estudo individual afetou significativamente as RPs agrupadas, indicando que nossos resultados eram estatisticamente robustos. Contudo, após excluir os dois estudos de caso-controle que não estavam de acordo com o EHW, tornando a amostra uma representação ruim, as RPs combinadas correspondentes foram materialmente alteradas em todos os modelos genéticos: alélico (C versus G: $R P=0,713$; IC95\%: 0,400-1,271; $p=0,251$ ), heterozigótico (CG versus GG: $R P=0,745$; IC95\%: $0,553-1,005 ; p=0,054)$, dominante $(C C+C G$ versus GG: RP $=0,701$; IC95\%: $0,377-1,302 ; p=0,261$ ) e recessivo (CC versus $C G+G G$ : $R P=0,490$; IC95\%: 0,170-1,409; $p=0,186$ ).

\section{Viés de Publicação}

O gráfico de funil de Begg e o teste de Egger foram realizados para avaliar o viés de publicação dos estudos incluídos. As formas dos gráficos de funil não revelaram nenhuma evidência de assimetria óbvia nos cinco modelos genéticos: alélico ( $C$ versus G: $p_{\text {Begg }}=0,754$ e $p_{\text {Egger }}=0,909$, - Fig. 3A), homozigótico (CC versus GG: $p_{\text {Begg }}=1,000$ e $p_{\text {Egger }}=0,792$ ), heterozigótico (CG versus GG: $p_{\text {Begg }}=0,754$ e $p_{\text {Egger }}=0,834$ ), 


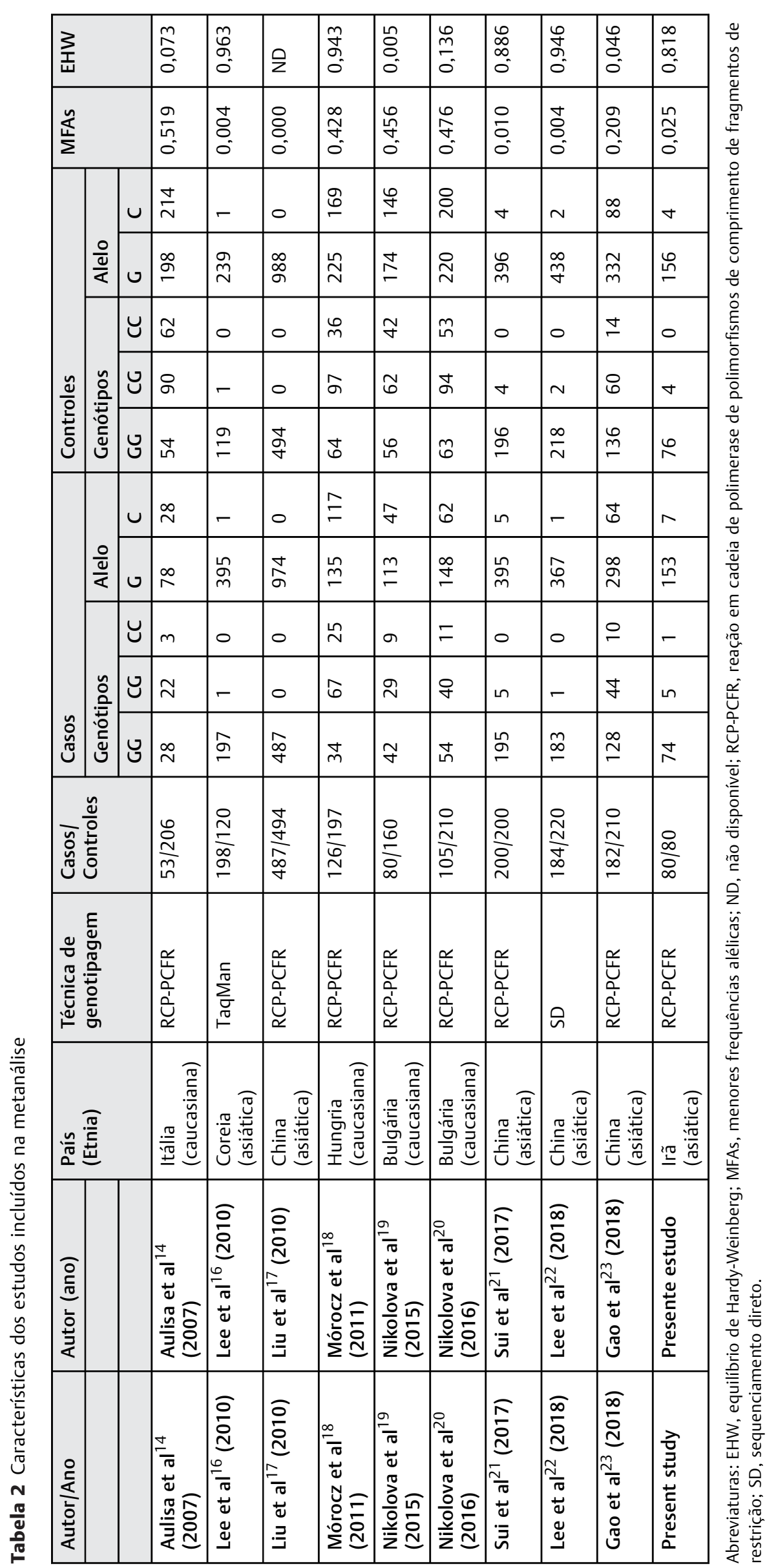


Tabela 3 Resultados da associação do polimorfismo do IL-6 -174 G > C com o risco de desenvolver EIA

\begin{tabular}{|c|c|c|c|c|c|c|c|c|c|c|}
\hline \multirow[t]{2}{*}{ Subgrupos } & \multirow[t]{2}{*}{$\begin{array}{l}\text { Modelo } \\
\text { genético }\end{array}$} & \multirow[t]{2}{*}{$\begin{array}{l}\text { Tipo de } \\
\text { modelo }\end{array}$} & \multicolumn{2}{|c|}{$\begin{array}{l}\text { Heterogenei- } \\
\text { dade }(\mathrm{H})\end{array}$} & \multicolumn{4}{|c|}{ Razão de probabilidades (RP) } & \multicolumn{2}{|c|}{$\begin{array}{l}\text { Viés de } \\
\text { Publicação }\end{array}$} \\
\hline & & & $I^{2}(\%)$ & $p_{\mathrm{H}}$ & $\mathrm{RP}$ & IC95\% & Teste $\mathbf{Z}$ & $p_{\mathrm{OR}}$ & $p_{\text {Begg }}$ & $p_{\text {Egger }}$ \\
\hline \multirow[t]{5}{*}{ Geral } & $C$ versus $G$ & Aleatório & 73,65 & $\leq 0,001$ & 0,671 & $0,457-0,985$ & $-2,035$ & 0,042 & 0,754 & 0,909 \\
\hline & CC versus GG & Aleatório & 77,57 & $\leq 0,001$ & 0,439 & $0,439-0,192$ & $-1,951$ & 0,051 & 1,000 & 0,792 \\
\hline & CG versus GG & Fixo & 22,24 & 0,245 & 0,734 & $0,554-0,973$ & $-2,150$ & 0,032 & 0,754 & 0,834 \\
\hline & $\mathrm{CC}+\mathrm{CG}$ versus $\mathrm{GG}$ & Aleatório & 61,46 & 0,008 & 0,660 & $0,440-0,990$ & $-2,010$ & 0,044 & 1,000 & 0,786 \\
\hline & CC versus CG + GG & Aleatório & 67,97 & 0,008 & 0,506 & $0,264-0,970$ & $-2,052$ & 0,040 & 1,000 & 0,727 \\
\hline \multicolumn{11}{|l|}{ Por etnia } \\
\hline \multirow[t]{5}{*}{ Asiática } & $C$ versus $G$ & Fixo & 0,00 & 0,816 & 0,865 & $0,624-1,201$ & $-0,866$ & 0,386 & 1,000 & 0,552 \\
\hline & CC versus GG & Fixo & 0,00 & 0,409 & 0,831 & $0,366-1,885$ & $-0,443$ & 0,658 & ND & ND \\
\hline & CG versus GG & Fixo & 0,00 & 0,915 & 0,840 & $0,562-1,256$ & $-0,851$ & 0,395 & 0,806 & 0,642 \\
\hline & $\mathrm{CC}+\mathrm{CG}$ versus GG & Fixo & 0,00 & 0,873 & 0,845 & $0,578-1,234$ & $-0,872$ & 0,383 & 1,000 & 0,571 \\
\hline & $\mathrm{CC}$ versus $\mathrm{CG}+\mathrm{GG}$ & Fixo & 0,00 & 0,437 & 0,885 & $0,394-1,989$ & $-0,296$ & 0,767 & ND & $\mathrm{ND}$ \\
\hline \multirow[t]{5}{*}{ Caucasiana } & $C$ versus $G$ & Aleatório & 88,10 & $\leq 0,001$ & 0,552 & $0,318-0,959$ & $-2,110$ & 0,035 & 0,308 & 0,173 \\
\hline & CC versus GG & Aleatório & 84,71 & $\leq 0,001$ & 0,329 & $0,114-0,951$ & $-2,053$ & 0,040 & 0,308 & 0,204 \\
\hline & CG versus GG & Aleatório & 65,43 & 0,034 & 0,670 & $0,413-1,086$ & $-1,626$ & 0,104 & 1,000 & 0,519 \\
\hline & $\mathrm{CC}+\mathrm{CG}$ versus $\mathrm{GG}$ & Aleatório & 81,71 & 0,001 & 0,541 & $0,290-1,008$ & $-1,935$ & 0,053 & 0,734 & 0,490 \\
\hline & CC versus CG + GG & Aleatório & 77,97 & 0,003 & 0,408 & $0,180-0,925$ & $-2,146$ & 0,032 & 0,308 & 0,114 \\
\hline \multirow{5}{*}{$\begin{array}{l}\text { Asiática } \\
\text { (Chinesa) }\end{array}$} & $C$ versus $G$ & Fixo & 0,00 & 0,790 & 0,824 & $0,585-1,160$ & $-1,109$ & 0,267 & 1,000 & 0,799 \\
\hline & CC versus GG & Fixo & 0,00 & 1,000 & 0,759 & $0,325-1,770$ & $-0,639$ & 0,523 & ND & ND \\
\hline & CG versus GG & Fixo & 0,00 & 0,775 & 0,811 & $0,530-1,243$ & $-0,961$ & 0,337 & 1,000 & 0,797 \\
\hline & $\mathrm{CC}+\mathrm{CG}$ versus $\mathrm{GG}$ & Fixo & 0,00 & 0,771 & 0,804 & $0,539-1,199$ & $-1,069$ & 0,285 & 1,000 & 0,780 \\
\hline & $C C$ versus $C G+G G$ & Fixo & 0,00 & 1,000 & 0,814 & $0,352-1,880$ & $-0,482$ & 0,630 & ND & ND \\
\hline
\end{tabular}

Abreviaturas: IC95\%, intervalo de confiança de 95\%; ND, não disponível.

dominante $\quad\left(\mathrm{CC}+\mathrm{CG}\right.$ versus $\mathrm{GG}: \quad p_{\text {Begg }}=1,000 \quad \mathrm{e}$ $p_{\text {Egger }}=0,786$, - Fig. $3 \mathbf{B}$ ) e recessivo (CC versus CG + GG: $p_{\text {Begg }}=1,000$ e $\left.p_{\text {Egger }}=0,727\right)$. 0 teste de Egger também não mostrou evidência estatística significativa de viés de publicação, o que indicou baixo risco disso nesta metaanálise.

\section{Menor Frequência Alélica}

A menor frequência alélica (MFA) do polimorfismo do IL-6 $-174 G>C$ nos controles saudáveis asiáticos e caucasianos é apresentada na - Tabela 2. As frequências geográficas do alelo IL-6 -174C foram de 10,45\% (0,00-20,9\%) entre asiáticos, e de $47,35 \%(42,80-51,90 \%)$ entre caucasianos, respectivamente (-Tabela 2 ).

\section{Discussão}

Acredita-se que fatores genéticos e epigenéticos tenham um papel importante na etiologia da EIA. ${ }^{2,24}$ No entanto, a relação entre os fatores de risco ambiental e a EIA pode ser altamente complicada, e uma extensa pesquisa é necessária para verificar como exatamente os fatores ambientais afetam a suscetibilidade individual a desenvolver EIA. ${ }^{1,2}$
Vários estudos de associação investigaram a associação entre o polimorfismo do IL-6 $-174 G>C$ e o risco de desenvolver EIA. Após reunir os dados de dez estudos elegíveis com 1.695 casos e 2.097 controles, descobrimos que a associação entre o polimorfismo do IL-6 $-174 \mathrm{G}>\mathrm{C}$ e o risco de EIA é estatisticamente significativa na população em geral. Uma análise estratificada adicional por etnia demonstrou uma associação significativa entre o polimorfismo do IL-6 -174G $>\mathrm{C}$ e o risco de desenvolver EIA entre caucasianos, mas não entre asiáticos. $\mathrm{O}$ resultado inconsistente entre os asiáticos na análise de subgrupos com as RPs gerais pode ser causado pela diversidade genética em diferentes etnias. Nossos resultados de metanálise foram consistentes com a metanálise anterior by Zhao et $\mathrm{al}^{15}$, baseada em 5 estudos de casocontrole com 944 casos e 1.177 controles. Em 2016, Zhao et $\mathrm{al}^{15}$ realizaram uma metanálise para avaliar a associação entre o polimorfismo do IL-6 -174G > C e o risco de desenvolver EIA. Seus resultados sugeriram que o polimorfismo do IL-6 -174G > C não tem influência significativa na suscetibilidade individual a desenvolver EIA. ${ }^{15}$ No entanto, essa metanálise incluía tamanho de amostra menor, e não conseguiu confirmar uma associação significativa. Portanto, a presente metanálise, com uma amostra maior, forneceu um 
24 Associação do polimorfismo IL-6 -174G > C (rs1800795) com escoliose idiopática da adolescência Sobhan et al.

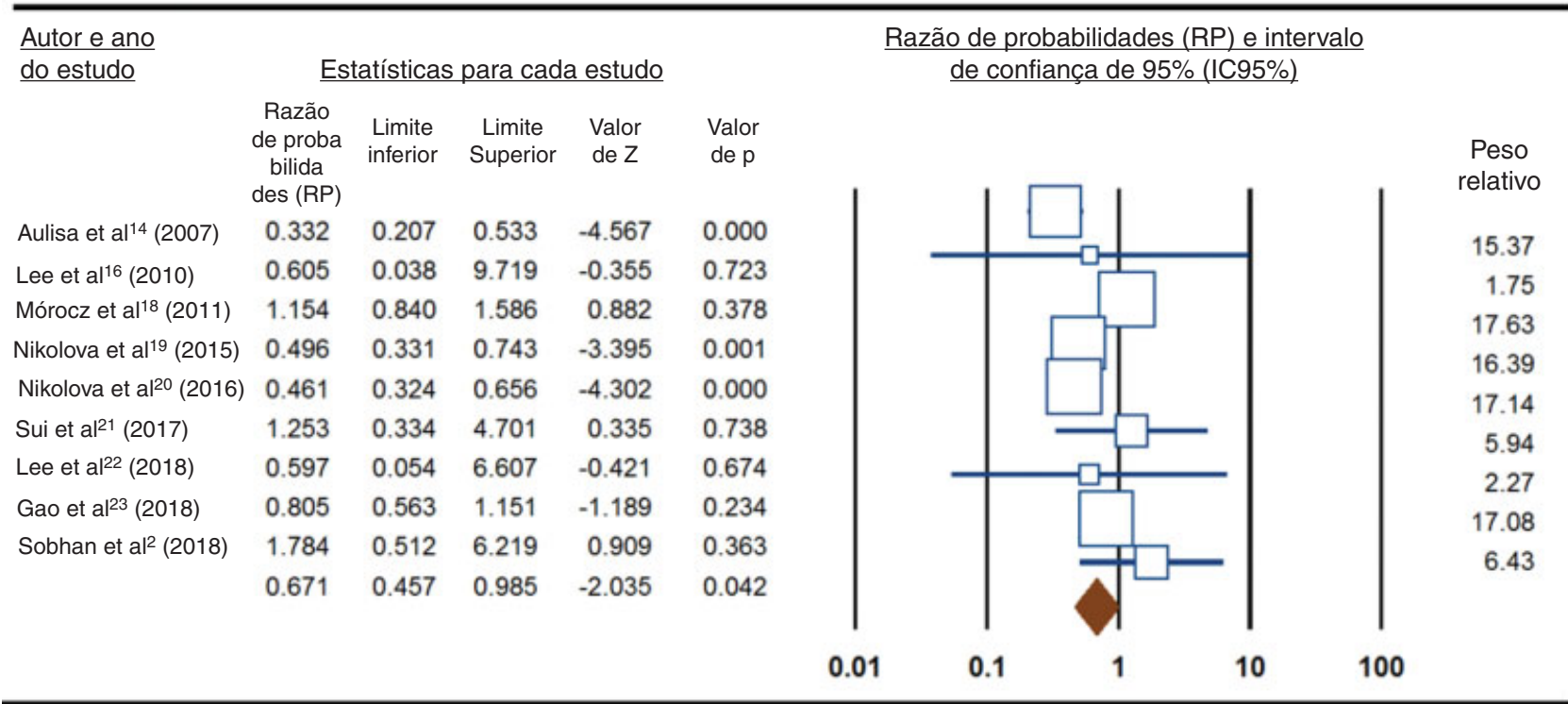

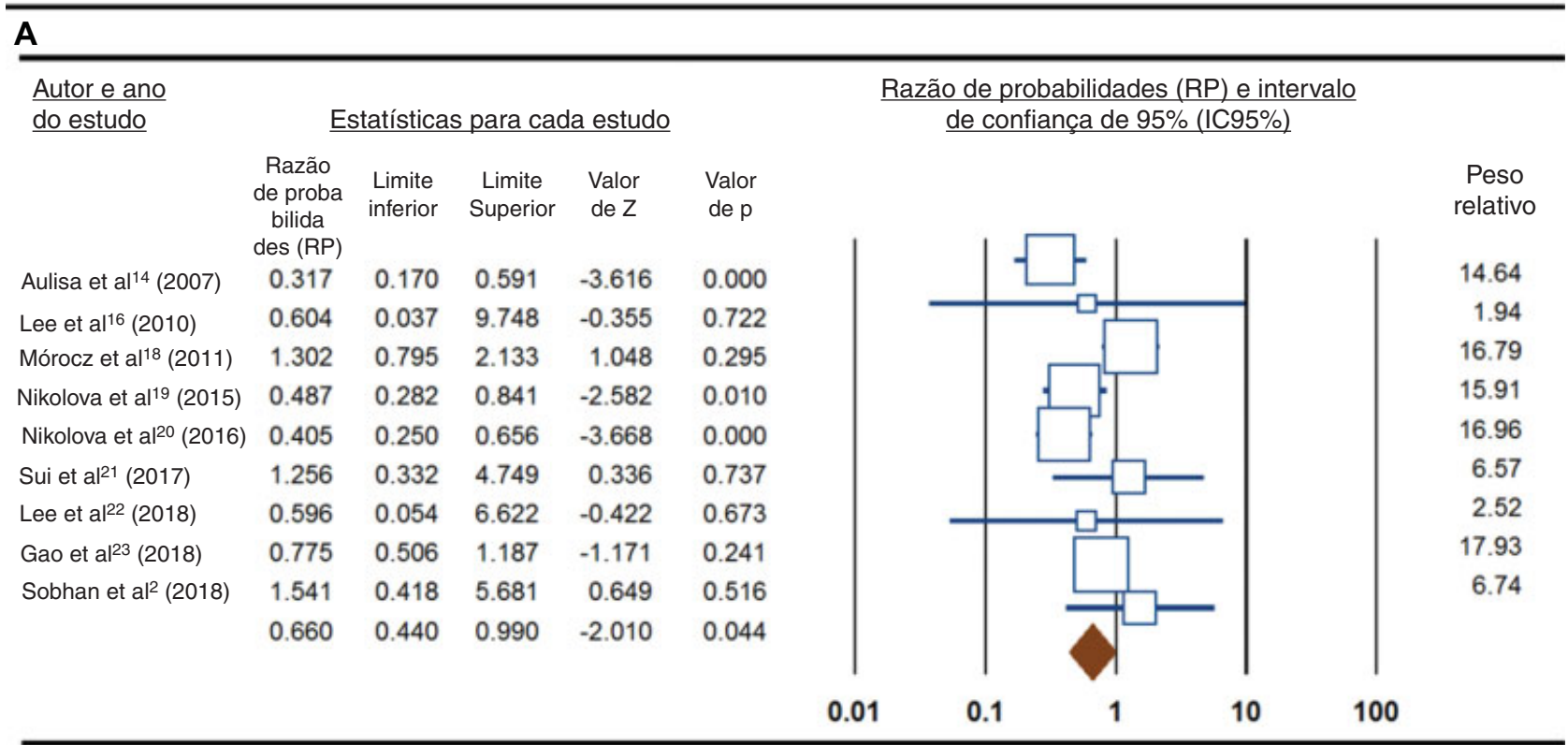

B

Fig. 2 Gráficos de floresta do polimorfismo do IL-6 -174G > C com risco de desenvolvimento de EIA. (A) Modelo alélico (C versus G); (B) modelo dominante (CC + CG versus GG). Foi utilizado um modelo de efeitos aleatórios.

resultado preciso em relação à associação do polimorfismo do IL-6 -174G >C com o risco de desenvolver EIA.

A heterogeneidade é um problema potencial que deve ser abordado, pois pode afetar os resultados de uma metanálise. ${ }^{25,26}$ A significativa heterogeneidade entre os estudos exibida pode ser atribuída à diferenças em vários fatores, como diferença de etnia, de tamanho da amostra, de técnicas de genotipagem, e diversidade no desenho e na execução dos estudos. ${ }^{27-30}$ No entanto, apesar de o número de estudos incluídos na presente metanálise ter sido pequeno, uma heterogeneidade relativamente grande foi observada em quatro modelos genéticos na população geral. Assim, realizamos uma análise de subgrupos por etnia para explorar as fontes de heterogeneidade. No entanto, após a estratificação por etnia, nenhuma heterogeneidade significativa foi observada na população asiática, mas a heterogeneidade ainda existia na população caucasiana. Portanto, pode-se presumir que a heterogeneidade relativamente grande resulta principalmente de origens étnicas.

Existem várias limitações em nossa metanálise. Primeiro, dado que apenas estudos publicados em inglês e chinês foram incluídos, pode haver viés de publicação, embora nossos resultados não tenham mostrado significância. Segundo, como os estudos incluídos foram conduzidos entre asiáticos e caucasianos, os resultados devem ser interpretados cuidadosamente. Mais estudos sobre populações de outras áreas, como África e América do Norte, são necessários para diminuir os vieses produzidos pela variação étnica. Terceiro, as análises atuais foram baseadas em estimativas não ajustadas, porque a maioria dos estudos não forneceu dados ajustados. Análises mais precisas, incluindo dados individuais, fatores de estilo de vida e ambientais, devem 

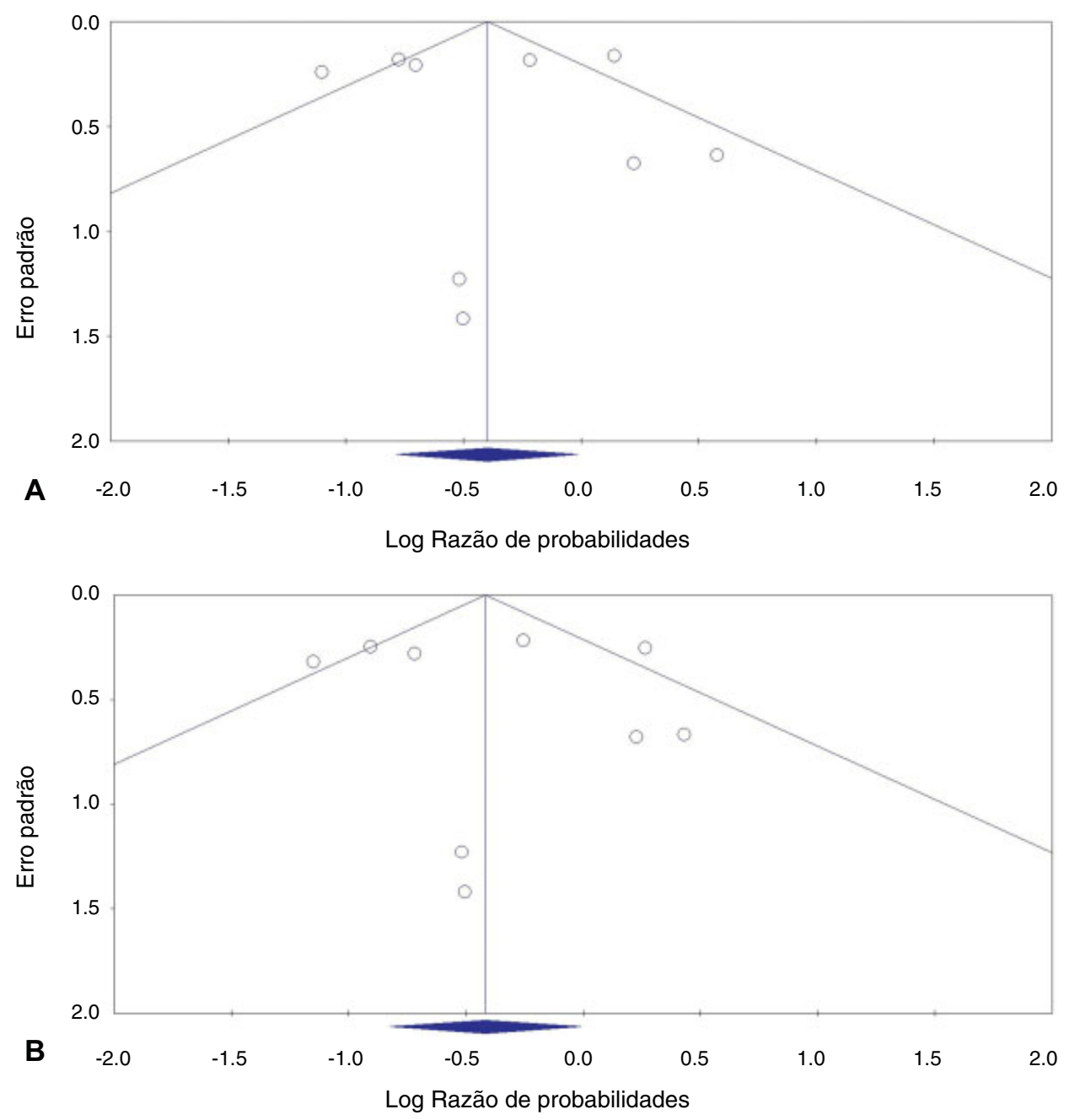

Fig. 3 Gráfico de funil para a detecção do viés de publicação para a associação do polimorfismo do IL-6 -174 G > C com o risco de desenvolver EIA. (A) Modelo alélico (C versus G); (B) modelo dominante (CC + CG versus GG). Um modelo de efeitos aleatórios foi utilizado.

ser realizadas, se possível. Finalmente, acredita-se que genética, meio ambiente e expossoma desempenhem um papel importante na fisiopatologia da EIA, mas a presente metanálise não pôde avaliar as interações gene-gene e geneambiente devido às informações limitadas dos estudos incluídos.

Em resumo, os resultados desta meta-análise, que são inconsistentes com os da metanálise anterior by Zhao et al ${ }^{15}$ indicam que o polimorfismo do IL-6 -174G > C está associado com o risco de desenvolver EIA, especialmente em caucasianos. Além disso, nosso estudo de caso-controle indicou que o polimorfismo do IL-6 $-174 \mathrm{G}>\mathrm{C}$ não estava associado com o risco de desenvolver EIA na população iraniana.

\section{Conflito de Interesses}

Os autores declaram não haver conflito de interesses.

\section{Referências}

1 Choudhry MN, Ahmad Z, Verma R. Adolescent Idiopathic Scoliosis. Open Orthop J 2016;10:143-154

2 Sobhan MR, Mahdinezhad-Yazdi M, Aghili K, et al. Association of TNF- $\alpha-308 \mathrm{G}>\mathrm{A}$ and $-238 \mathrm{G}>\mathrm{A}$ polymorphisms with knee osteoarthritis risk: A case-control study and meta-analysis. J Orthop 2018;15(03):747-753

3 Beauséjour M, Goulet L, Parent S, et al. Members of the Quebec Scoliosis Society and of the Canadian Paediatric Spinal Deformities Study Group. The effectiveness of scoliosis screening programs: methods for systematic review and expert panel recommendations formulation. Scoliosis 2013;8(01):12

4 Illés T, Tunyogi-Csapó M, Somoskeöy S. Breakthrough in threedimensional scoliosis diagnosis: significance of horizontal plane view and vertebra vectors. Eur Spine J 2011;20(01):135-143

5 Wu H, Ronsky JL, Cheriet F, Harder J, Küpper JC, Zernicke RF. Time series spinal radiographs as prognostic factors for scoliosis and progression of spinal deformities. Eur Spine J 2011;20(01):112-117

6 Daryabor A, Arazpour M, Sharifi G, Bani MA, Aboutorabi A, Golchin N. Gait and energy consumption in adolescent idiopathic scoliosis: A literature review. Ann Phys Rehabil Med 2017;60(02): 107-116

7 Zheng X, Wang W, Qian B, et al. Accelerated endochondral growth in adolescents with idiopathic scoliosis: a preliminary histomorphometric study. BMC Musculoskelet Disord 2014;15(01):429

8 Paria N, Wise CA. Genetics of adolescent idiopathic scoliosis. Semin Spine Surg 2015;27(01):9-15

9 Ikegawa S. Genomic study of adolescent idiopathic scoliosis in Japan. Scoliosis Spinal Disord 2016;11(01):5

10 Dai J, Lv ZT, Huang JM, Cheng P, Fang H, Chen AM. Association between polymorphisms in vitamin $\mathrm{D}$ receptor gene and 
26 Associação do polimorfismo IL-6 -174G > C (rs1800795) com escoliose idiopática da adolescência Sobhan et al.

adolescent idiopathic scoliosis: a meta-analysis. Eur Spine J 2018; 27(09):2175-2183

11 Xu L, Sheng F, Xia C, et al. Common Variant of POC5 Is Associated With the Susceptibility of Adolescent Idiopathic Scoliosis. Spine 2018;43(12):E683-E688

12 Tanaka T, Narazaki M, KishimotoT. IL-6 in inflammation, immunity, and disease. Cold Spring Harb Perspect Biol 2014;6(10):a016295

13 Popko K, Gorska E, Demkow U. Influence of interleukin-6 and G174C polymorphism in IL-6 gene on obesity and energy balance. Eur J Med Res 2010;15(Suppl 2):123-127

14 Aulisa L, Papaleo P, Pola E, et al. Association between IL-6 and MMP-3 gene polymorphisms and adolescent idiopathic scoliosis: a case-control study. Spine 2007;32(24):2700-2702

15 Zhao J, Yang M, Li M. Association of IL-6 and MMP-3 gene polymorphisms with susceptibility to adolescent idiopathic scoliosis: a meta-analysis. J Genet 2016;95(03):573-579

16 Lee JS, Suh KT, Eun IS. Polymorphism in interleukin-6 gene is associated with bone mineral density in patients with adolescent idiopathic scoliosis. J Bone Joint Surg Br 2010;92(08):1118-1122

17 Liu Z, Tang NL, Cao XB, et al. Lack of association between the promoter polymorphisms of MMP-3 and IL- 6 genes and adolescent idiopathic scoliosis: a case-control study in a Chinese Han population. Spine 2010;35(18):1701-1705

18 Mórocz M, Czibula A, Grózer ZB, et al. Association study of BMP4, IL6, Leptin, MMP3, and MTNR1B gene promoter polymorphisms and adolescent idiopathic scoliosis. Spine 2011;36(02):E123-E130

19 Nikolova S, Dikova M, Dikov D, et al. Role of the IL-6 gene in the etiopathogenesis of idiopathic scoliosis. Anal Cell Pathol (Amst) 2015;2015:621893

20 Nikolova ST, Yablanski VT, Vlaev EN, et al. Association Between IL6 and MMP3 Common Genetic Polymorphisms and Idiopathic Scoliosis in Bulgarian Patients: A Case-control Study. Spine 2016; 41(09):785-791

21 Sui W, Yang J, Huang Z, Wang Q, Fan H, Deng Y. Polymorphisms in promoter regions of MMP-3 and IL- 6 genes are not associated to adolescent idiopathic scoliosis (AIS) gender bias. JBack Musculoskeletal Rehabil 2017;30(03):559-563

22 Lee JS, Shin JK, Goh TS. Interleukin 6 gene polymorphism in patients with degenerative lumbar scoliosis: a cohort study. Eur Spine J 2018;27(03):607-612
23 Gao J, Zhang L, Liu Z, Yao S, Gao S. [Correlation analysis between interleukin 6 polymorphism and adolescent idiopathic scoliosis susceptibility and bracing effectiveness]. Zhongguo Xiu Fu Chong Jian Wai Ke Za Zhi 2018;32(06):678-684

24 Burwell RG, Dangerfield PH, Moulton A, Grivas TB. Adolescent idiopathic scoliosis (AIS), environment, exposome and epigenetics: a molecular perspective of postnatal normal spinal growth and the etiopathogenesis of AIS with consideration of a network approach and possible implications for medical therapy. Scoliosis 2011;6(01):26

25 Jafari Nedooshan J, Kargar S, Neamatzadeh H, Haghighi F, Dehghani Mohammad Abadi R, Seddighi N. Lack of Association of the Fat Mass and Obesity Associated (FTO) Gene rs9939609 Polymorphism with Breast Cancer Risk: a Systematic Review and MetaAnalysis Based on Case - Control Studies. Asian Pac J Cancer Prev 2017;18(04):1031-1037

26 Sadeghiyeh T, Hosseini Biouki F, Mazaheri M, Zare-Shehneh M, Neamatzadeh H, Poursharif Z. Association between CatecholO-Methyltransferase Val158Met (158G/A) Polymorphism and Suicide Susceptibility: A Meta-analysis. J Res Health Sci 2017; 17(02):e00383

27 Abedinzadeh M, Zare-Shehneh M, Neamatzadeh H, Abedinzadeh M, Karami H. Association between MTHFR C677T polymorphism and risk of prostate cancer: Evidence from 22 studies with 10,832 cases and 11,993 controls. Asian Pac J Cancer Prev 2015;16(11): 4525-4530

28 Yazdi MM, Jamalaldini MH, Sobhan MR, et al. Association of ESR $\alpha$ Gene Pvu II T>C, XbaI A>G and BtgI G $>$ A Polymorphisms with Knee Osteoarthritis Susceptibility: A Systematic Review and Meta-Analysis Based on 22 Case-Control Studies. Arch Bone Jt Surg 2017;5(06):351-362

29 Kamali M, Hamadani S, Neamatzadeh $\mathrm{H}$, et al. Association of XRCC2 rs3218536 polymorphism with susceptibility of breast and ovarian cancer: A systematic review and meta-analysis. Asian Pac J Cancer Prev 2017;18(07):1743-1749

30 Gohari M, Neámatzadeh H, Jafari MA, Mazaheri M, Zare-Shehneh M, Abbasi-Shavazi E. Association between the p53 codon 72 polymorphism and primary open-angle glaucoma risk: Metaanalysis based on 11 case-control studies. Indian J Ophthalmol 2016;64(10):756-761 